\title{
A Limit Relation for Dunkl-Bessel Functions of Type A and $B^{\star}$
}

Margit RÖSLER ${ }^{\dagger}$ and Michael VOIT ${ }^{\ddagger}$

$\dagger$ Institut für Mathematik, TU Clausthal, Erzstr. 1, D-38678 Clausthal-Zellerfeld, Germany

E-mail: roesler@math.tu-clausthal.de

$\ddagger$ Fachbereich Mathematik, TU Dortmund, Vogelpothsweg 87, D-44221 Dortmund, Germany

E-mail: michael.voit@math.tu-dortmund.de

Received October 21, 2008, in final form November 26, 2008; Published online December 03, 2008

Original article is available at http://www.emis.de/journals/SIGMA/2008/083/

Abstract. We prove a limit relation for the Dunkl-Bessel function of type $B_{N}$ with mul-
tiplicity parameters $k_{1}$ on the roots $\pm e_{i}$ and $k_{2}$ on $\pm e_{i} \pm e_{j}$ where $k_{1}$ tends to infinity and
the arguments are suitably scaled. It gives a good approximation in terms of the Dunkl-
type Bessel function of type $A_{N-1}$ with multiplicity $k_{2}$. For certain values of $k_{2}$ an improved
estimate is obtained from a corresponding limit relation for Bessel functions on matrix cones.

Key words: Bessel functions; Dunkl operators; asymptotics

2000 Mathematics Subject Classification: 33C67; 43A85; 20F55

\section{Introduction and results}

The power series expansion

$$
j_{\alpha}(z)=\Gamma(\alpha+1) \sum_{n=0}^{\infty} \frac{(-1)^{n}(z / 2)^{2 n}}{n ! \Gamma(n+\alpha+1)}
$$

of the normalized spherical Bessel functions $j_{\alpha}(z)={ }_{0} F_{1}\left(\alpha+1 ;-z^{2} / 4\right)$ leads immediately to the well known limit relation

$$
\lim _{\alpha \rightarrow \infty} j_{\alpha}(\sqrt{\alpha} \cdot z)=e^{-z^{2} / 4} \quad \text { for } \quad z \in \mathbb{C} .
$$

For real arguments, this limit relation can be improved as follows: There exists a constant $C>0$ such that

$$
\left|j_{\mu-1}(\sqrt{\mu} \cdot x)-e^{-x^{2} / 4}\right| \leq \frac{C}{\mu} \cdot \min \left(x^{4}, 1\right) \quad \text { for all } \quad x \in \mathbb{R}, \mu>2 .
$$

This is the rank-one specialization of a more general result for Bessel functions on cones of positive semidefinite matrices obtained in [14, Theorem 3.6]. For related asymptotic results for one-variable Bessel functions $j_{\alpha}$ as $\alpha \rightarrow \infty$ we refer to [17]. In [16], a variant of estimate (1) was used to derive a law of large numbers for radial random walks on $\mathbb{R}^{p}$ where the time parameter of the walks as well as the dimension $p$ tend to infinity. In this case, Bessel functions of index $\alpha=\frac{p}{2}-1$ come in as the radial parts of the complex exponential functions, and estimate (1) leads to limit theorems for radial random walks. This approach was extended in [14] to radial random walks on matrix spaces over one of the (skew-) fields $\mathbb{F}=\mathbb{R}, \mathbb{C}, \mathbb{H}$.

\footnotetext{
${ }^{\star}$ This paper is a contribution to the Special Issue on Dunkl Operators and Related Topics. The full collection is available at http://www.emis.de/journals/SIGMA/Dunkl_operators.html
} 
In the present note we derive a further multidimensional extension of (1), namely for certain Bessel functions of Dunkl-type; see [3, 11] for an introduction to Dunkl theory and [10] for the associated Bessel functions. More precisely, we shall prove that under suitable normalization of the arguments, the Bessel function $J_{\left(k_{1}, k_{2}\right)}^{B}$ of type $B_{q}$ converges to the Bessel function $J_{k_{2}}^{A}$ of type $A_{q-1}$ where the multiplicity parameter $k_{2}$ (on the roots $\pm e_{i} \pm e_{j}$ ) is fixed and $k_{1}$ (on the roots $\pm e_{i}$ ) tends to infinity. The obtained estimate is optimal for small arguments, but for large arguments it is weaker than (1) and the corresponding result in [14] for Bessel functions of matrix argument. This is due to the fact that the proofs of (1) and its matrix version in [14] rely on some explicit integral representation of the Bessel functions which is as far not available for Dunkl-type Bessel functions in general. Nevertheless, our limit result should be of some interest in its own. In the following, we state our limit results. Proofs will be given in Sections 2 and 3.

We first recapitulate the necessary facts from Dunkl theory. We shall not go into details but refer the reader to [3, 11] and [14] for more background. For multivariable hypergeometric functions, see e.g. [1,5] and [8]. For a reduced root system $R \subset \mathbb{R}^{N}$ and a multiplicity function $k: R \rightarrow[0, \infty)$ (i.e. $k$ is invariant under the action of the corresponding reflection group), we denote by $E_{k}$ the corresponding Dunkl kernel and by $J_{k}$ the Bessel function associated with $R$ and $k$ which is given by

$$
J_{k}(x, y)=\frac{1}{|W|} \sum_{w \in W} E_{k}(w x, y)
$$

where the sum is over the underlying reflection group $W$. Bessel functions associated with root systems generalize the spherical functions of flat symmetric spaces which occur for crystallographic root systems and specific discrete values of $k$. We shall be concerned with Bessel functions associated with root systems of type $A$ and $B$ which can be expressed in terms of Jack polynomial series. To be precise, let $C_{\lambda}^{\alpha}$ denote the Jack polynomials of index $\alpha>0$ which are indexed by partitions $\lambda=\left(\lambda_{1} \geq \cdots \geq \lambda_{N}\right) \in \mathbb{N}_{0}^{N}$, see [15]. The $C_{\lambda}^{\alpha}$ are homogeneous of degree $|\lambda|=\lambda_{1}+\cdots+\lambda_{N}$ and can be normalized such that

$$
\left(x_{1}+\cdots+x_{N}\right)^{k}=\sum_{|\lambda|=k} C_{\lambda}^{\alpha}(x) \quad \text { for all } \quad k \in \mathbb{N}_{0} ;
$$

this normalization will be adopted here. For root system $A_{N-1}=\left\{ \pm\left(e_{i}-e_{j}\right): i<j\right\} \subset \mathbb{R}^{N}$, the multiplicity $k$ is a single real parameter. Due to relations (3.22) and (3.37) of [2], the associated Bessel function can be expressed as a generalized ${ }_{0} F_{0}$-hypergeometric function,

$$
J_{k}^{A}(x, y)={ }_{0} F_{0}^{\alpha}(x, y):=\sum_{\lambda \geq 0} \frac{1}{|\lambda| !} \cdot \frac{C_{\lambda}^{\alpha}(x) C_{\lambda}^{\alpha}(y)}{C_{\lambda}^{\alpha}(\mathbf{1})} \quad \text { with } \quad \mathbf{1}=(1, \ldots, 1), \quad \alpha=1 / k,
$$

where $\lambda \geq 0$ denotes that the sum is taken over all partitions $\lambda=\left(\lambda_{1} \geq \cdots \geq \lambda_{N}\right)$. For root system $B_{N}=\left\{ \pm e_{i}, \pm e_{i} \pm e_{j}: i<j\right\}$, the multiplicity is of the form $k=\left(k_{1}, k_{2}\right)$ where $k_{1}$ and $k_{2}$ are the values on the roots $\pm e_{i}$ and $\pm e_{i} \pm e_{j}$ respectively. The associated Bessel function is given by

$$
J_{k}^{B}(x, y)={ }_{0} F_{1}^{\alpha}\left(\mu ; \frac{x^{2}}{2}, \frac{y^{2}}{2}\right) \quad \text { with } \quad \alpha=\frac{1}{k_{2}}, \quad \mu=k_{1}+(N-1) k_{2}+\frac{1}{2},
$$

where $x^{2}:=\left(x_{1}^{2}, \ldots, x_{N}^{2}\right)$ and

$$
{ }_{0} F_{1}^{\alpha}(\mu ; x, y):=\sum_{\lambda \geq 0} \frac{1}{(\mu)_{\lambda}^{\alpha}|\lambda| !} \cdot \frac{C_{\lambda}^{\alpha}(x) C_{\lambda}^{\alpha}(y)}{C_{\lambda}^{\alpha}(\mathbf{1})} .
$$

We denote by $\langle\cdot, \cdot\rangle$ and $|\cdot|$ the usual Euclidean scalar product and norm on $\mathbb{R}^{N}$. The main results of this note are as follows: 
Proposition 1. Let $N \geq 2$ and $k_{2} \geq 0$. Then there exists a constant $C=C\left(N, k_{2}\right)>0$ such that for all $k_{1} \geq k_{2}(N-1), x, y \in \mathbb{R}^{N}$, and $\mu=k_{1}+k_{2}(N-1)+\frac{1}{2}$,

$$
\left|J_{\left(k_{1}, k_{2}\right)}^{B}(2 \sqrt{\mu} x, i y)-J_{k_{2}}^{A}\left(-x^{2}, y^{2}\right)\right| \leq \frac{C}{\mu} \cdot|x|^{4}|y|^{4} \cdot e^{|x|^{2}|y|^{2}} .
$$

For certain values of $k_{2}$, this estimate can be improved as follows:

Proposition 2. Let $N \geq 2$ and $k_{2} \in\left\{0, \frac{1}{2}, 1,2\right\}$. Then there exists a constant $C=C\left(N, k_{2}\right)>0$ such that for all $k_{1} \geq k_{2}(N-1), x, y \in \mathbb{R}^{N}$, and $\mu$ as above,

$$
\left|J_{\left(k_{1}, k_{2}\right)}^{B}(2 \sqrt{\mu} x, i y)-J_{k_{2}}^{A}\left(-x^{2}, y^{2}\right)\right| \leq \frac{C}{\mu} \cdot \min \left(|x|^{4}|y|^{4}, 1\right) .
$$

In contrast to the previous estimate which is only locally uniform, this estimate is uniform in $x$ and $y$. For $x, y$ close to 0 , it gives the same rate of convergence for $\mu \rightarrow \infty$. We conjecture that Proposition 2 is actually correct for all $k_{2} \geq 0$, and that a similar estimate is valid for the associated Dunkl kernels of type $A$ and type $B$.

\section{Proof of Proposition 2}

\subsection{The case $k_{2}=0$}

The argumentation in this case is different from that in the remaining cases and based on a reduction to the rank one case. Indeed, the Dunkl operators of type B with multiplicity $\left(k_{1}, 0\right)$ may be regarded as Dunkl operators for the reflection group $\mathbb{Z}_{2}^{N}$ and multiplicity $k_{1}=: k$. Thus the Dunkl kernel $E_{(k, 0)}^{B}$ factorizes as $E_{(k, 0)}^{B}(x, y)=\prod_{l=1}^{N} E_{k}^{\mathbb{Z}_{2}}\left(x_{l}, y_{l}\right)$ and the associated Bessel function is given by

$$
J_{(k, 0)}^{B}(x, y)=\frac{1}{N !} \sum_{w \in S_{N}} G_{k}(w x, y), \quad \text { where } \quad G_{k}(x, y)=\prod_{l=1}^{N} J_{k}^{\mathbb{Z}_{2}}\left(x_{l}, y_{l}\right)
$$

and $J_{k}^{\mathbb{Z}_{2}}$ is the Bessel function for root system $\mathbb{Z}_{2}$ on $\mathbb{R}$, that is $J_{k}^{\mathbb{Z}_{2}}(x, y)=j_{k-\frac{1}{2}}(i x y)$. On the other hand, the type $A$ Bessel function with multiplicity 0 is just

$$
J_{0}^{A}(x, y)=\frac{1}{N !} \sum_{w \in S_{N}} e^{\langle w x, y\rangle} .
$$

Thus

$$
\left|J_{(k, 0)}^{B}(2 \sqrt{\mu} x, i y)-J_{0}^{A}\left(-x^{2}, y^{2}\right)\right| \leq \frac{1}{N !} \sum_{w \in S_{N}}\left|G_{k}(2 \sqrt{\mu} w x, i y)-e^{-\left\langle(w x)^{2}, y^{2}\right\rangle}\right| .
$$

Further, for $x, y \in \mathbb{R}^{N}$,

$$
\begin{aligned}
\left|G_{k}(2 \sqrt{\mu} x, i y)-e^{-\left\langle x^{2}, y^{2}\right\rangle}\right|= & \left|\prod_{l=1}^{N} j_{k-\frac{1}{2}}\left(2 \sqrt{\mu} x_{l} y_{l}\right)-\prod_{l=1}^{N} e^{-x_{l}^{2} y_{l}^{2}}\right| \\
& \leq \sum_{l=1}^{N}\left|j_{k-\frac{1}{2}}\left(2 \sqrt{\mu} x_{l} y_{l}\right)-e^{-x_{l}^{2} y_{l}^{2}}\right|,
\end{aligned}
$$

where $\mu=k+\frac{1}{2}$ and the last inequality is obtained by a telescope argument and the fact that the factors in both products are bounded by 1 . By (1), the last sum can be estimated by $\frac{C^{\prime}}{\mu} \cdot \min \left(1,|x|^{4}|y|^{4}\right)$, and this yields the stated result. 


\subsection{The cases $k_{2}=\frac{1}{2}, 1,2$}

In these cases, the Bessel functions of type $\mathrm{B}$ are closely related with Bessel functions on the matrix cones $\Pi_{N}=\Pi_{N}(\mathbb{F})$ of positive semidefinite $N \times N$ matrices over $\mathbb{F}=\mathbb{R}, \mathbb{C}, \mathbb{H}$ as explained in [12]. Using this connection, we shall derive Proposition 2 from a corresponding result for Bessel functions on matrix cones in [14].

We first recapitulate some facts about Bessel functions of matrix argument. Fix one of the skew-fields $\mathbb{F}=\mathbb{R}, \mathbb{C}, \mathbb{H}$ with real dimension $d=1,2,4$, respectively. The Bessel functions associated with the cone $\Pi_{N}=\Pi_{N}(\mathbb{F})$ are defined in terms of its spherical polynomials which are indexed by partitions $\lambda=\left(\lambda_{1} \geq \cdots \geq \lambda_{N}\right) \in \mathbb{N}_{0}^{N}$ and given by

$$
\Phi_{\lambda}(X)=\int_{U_{N}} \Delta_{\lambda}\left(U X U^{-1}\right) d U
$$

where $d U$ is the normalized Haar measure of the unitary group $U_{N}=U_{N}(\mathbb{F})$ and $\Delta_{\lambda}$ denotes the power function

$$
\Delta_{\lambda}(X):=\Delta_{1}(X)^{\lambda_{1}-\lambda_{2}} \Delta_{2}(X)^{\lambda_{2}-\lambda_{3}} \cdots \Delta_{N}(X)^{\lambda_{N}}
$$

on the vector space $H_{N}=\left\{X \in M_{N}(\mathbb{F}): X=X^{*}\right\}$ of Hermitian $N \times N$ matrices over $\mathbb{F}$. The $\Delta_{i}(X)$ are the principal minors of the determinant $\Delta(X)$, see [4] for details. There is a renormalization $Z_{\lambda}=c_{\lambda} \Phi_{\lambda}$ with constants $c_{\lambda}>0$ depending on $\Pi_{N}$ such that

$$
(\operatorname{tr} X)^{k}=\sum_{|\lambda|=k} Z_{\lambda}(X) \quad \text { for all } \quad k \in \mathbb{N}_{0},
$$

see Section XI.5 of [4]. By construction, the $Z_{\lambda}$ are invariant under conjugation by $U_{N}$ and thus depend only on the eigenvalues of their argument. More precisely, for $X \in H_{N}$ with eigenvalues $x=\left(x_{1}, \ldots, x_{N}\right) \in \mathbb{R}^{N}$,

$$
Z_{\lambda}(X)=C_{\lambda}^{\alpha}(x) \quad \text { with } \quad \alpha=\frac{2}{d},
$$

where the $C_{\lambda}^{\alpha}$ are the Jack polynomials of index $\alpha$ (cf. $\left.[4,8,13]\right)$.

The Bessel functions on the cone $\Pi_{N}$ are defined as ${ }_{0} F_{1}$-hypergeometric series in terms of the $Z_{\lambda}$, namely

$$
J_{\mu}(X)=\sum_{\lambda \geq 0} \frac{(-1)^{|\lambda|}}{(\mu)_{\lambda}|\lambda| !} Z_{\lambda}(X)
$$

where the sum is over all partitions $\lambda=\left(\lambda_{1} \geq \cdots \geq \lambda_{N}\right) \in \mathbb{N}_{0}^{N}$ and $(\mu)_{\lambda}$ denotes the generalized Pochhammer symbol

$$
(\mu)_{\lambda}=(\mu)_{\lambda}^{2 / d} \quad \text { where } \quad(\mu)_{\lambda}^{\alpha}:=\prod_{j=1}^{N}\left(\mu-\frac{1}{\alpha}(j-1)\right)_{\lambda_{j}} \quad(\alpha>0) .
$$

In (5), the index $\mu \in \mathbb{C}$ is supposed to satisfy $(\mu)_{\lambda}^{\alpha} \neq 0$ for all $\lambda \geq 0$. If $N=1$, then $\Pi_{1}=\mathbb{R}_{+}$and the Bessel function $\mathcal{J}_{\mu}$ is independent of $d$ and given by a usual one-variable Bessel function,

$$
J_{\mu}\left(\frac{x^{2}}{4}\right)=j_{\mu-1}(x)
$$

There exist commutative convolution algebras (so-called hypergroup structures) on the cone $\Pi_{N}$ with convolutions which depend on the parameter $\mu$ and which have the Bessel functions 
$\varphi_{Y}(X)=\mathcal{J}_{\mu}\left(\frac{1}{4} Y X^{2} Y\right), Y \in \Pi_{N}$, as characters. For details we refer to [12]. Moreover, the unitary group $U_{N}$ acts by the usual conjugation $X \mapsto U X U^{-1}$ on $\Pi_{N}$ as a compact group of hypergroup automorphisms, i.e., these conjugations preserve these convolution structures. As shown in [12], this observation induces a further commutative hypergroup structure on the associatd orbit space $\Pi_{N}^{U_{N}}$ where this space may obviously be identified with the the set of all possible eigenvalues of matrices from $\Pi_{N}$ ordered by size, i.e. on the $B_{N}$-Weyl chamber

$$
\Xi_{N}=\left\{x=\left(x_{1}, \ldots, x_{N}\right) \in \mathbb{R}^{N}: x_{1} \geq \cdots \geq x_{N} \geq 0\right\} .
$$

The characters of this hypergroup are given by the $U_{N}$-means

$$
\psi_{y}(x)=\int_{U_{N}} \mathcal{J}_{\mu}\left(\frac{1}{4} y U x^{2} U^{-1} y\right) d U=J_{k(\mu, d)}^{B}(x, i y), \quad x, y \in \Xi_{N},
$$

where

$$
k(\mu, d):=(\mu-(d(N-1)+1) / 2, d / 2)
$$

and elements from $\Xi_{N}$ are identified with diagonal matrices in the natural way. For details, see Section 4 of [12]. We shall now deduce the claimed estimate for $J_{k}^{B}$ from the estimate for the Bessel functions $J_{\mu}$ on the cone $\Pi_{N}$ mentioned in the introduction. Indeed, according to Theorem 3.6 of [14] there exists a constant $C=C(N, d)>0$ such that for all $\mu>d(2 N-1)+1$ and $X \in \Pi_{N}$,

$$
\left|J_{\mu}(\mu X)-e^{-\operatorname{tr} X}\right| \leq \frac{C}{\mu} \cdot \min \left(1,(\operatorname{tr} X)^{2}\right) .
$$

In view of (6), this leads to the following estimate for the Dunkl-type Bessel function $J_{k(\mu, d)}^{B}$ :

$$
\left|J_{k(\mu, d)}^{B}(2 \sqrt{\mu} x, i y)-\int_{U_{N}} e^{-\operatorname{tr}\left(y U x^{2} U^{-1} y\right)} d U\right| \leq \frac{C}{\mu} \cdot \min (1, S(x, y)),
$$

where

$$
S(x, y)=\int_{U_{N}}\left|\operatorname{tr}\left(y U x^{2} U^{-1} y\right)\right|^{2} d U \leq \int_{U_{N}} \operatorname{tr}\left(y^{4}\right) \cdot \operatorname{tr}\left(U x^{4} U^{-1}\right) d U=\left|x^{2}\right|^{2}\left|y^{2}\right|^{2} \leq|x|^{4}|y|^{4} .
$$

On the other hand, the Jack polynomials $C_{\lambda}^{\alpha}$ with $\alpha=2 / d$ satisfy the product formula

$$
\frac{C_{\lambda}^{\alpha}\left(x^{2}\right) C_{\lambda}^{\alpha}\left(y^{2}\right)}{C_{\lambda}^{\alpha}(\mathbf{1})}=\int_{U_{N}} C_{\lambda}^{\alpha}\left(y U x^{2} U^{-1} y\right) d U \quad \text { for } \quad x, y \in \Xi_{N} .
$$

This follows from a corresponding product formula for the spherical polynomials, see Proposition 5.5 of [5]. Thus by equation (2) we further obtain, with $\alpha=2 / d$,

$$
\begin{aligned}
\int_{U_{N}} e^{-\operatorname{tr}\left(y U X^{2} U^{-1} y\right)} d U & =\sum_{\lambda \geq 0} \frac{1}{|\lambda| !} \int_{U_{N}} C_{\lambda}^{\alpha}\left(-y U x^{2} U^{-1} y\right) d U \\
& =\sum_{\lambda \geq 0} \frac{1}{|\lambda| !} \frac{C_{\lambda}^{\alpha}\left(-x^{2}\right) C_{\lambda}^{\alpha}\left(y^{2}\right)}{C_{\lambda}^{\alpha}(\mathbf{1})}={ }_{0} F_{0}^{\alpha}\left(-x^{2}, y^{2}\right),
\end{aligned}
$$

which implies the assertion.

Remark 1. The integral on the left side of formula (7) is of Harish-Chandra type. If $\mathbb{F}=\mathbb{C}$, then by Theorem II.5.35 of [6] it can be written as an alternating sum

$$
\int_{U_{N}} e^{-\operatorname{tr}\left(y U x^{2} U^{-1} y\right)} d U=\frac{\prod_{j=1}^{N-1} j !}{\pi\left(x^{2}\right) \pi\left(y^{2}\right)} \sum_{w \in S_{N}} \operatorname{sgn}(w) e^{-\left\langle x^{2}, w y^{2}\right\rangle},
$$

where $\pi(x)=\prod_{i<j}\left(x_{i}-x_{j}\right)$ is the fundamental alternating polynomial. 


\section{Proof of Proposition 1}

We now turn to the proof of Proposition 1 which is based on the power series representations (3) and (4) for the Bessel functions of type A and B. The proof is similar to the corresponding result for Bessel functions on matrix cones in [14]. We start with an observation about Jack polynomials.

Lemma 1. For all $x, y \in \mathbb{R}^{N}, m \in \mathbb{N}$, and $\alpha>0$,

$$
\sum_{\lambda ;|\lambda|=m} \frac{C_{\lambda}^{\alpha}\left(x^{2}\right) C_{\lambda}^{\alpha}\left(y^{2}\right)}{C_{\lambda}^{\alpha}(\mathbf{1})} \leq|x|^{2 m}|y|^{2 m} .
$$

Proof. As shown in [9], the $C_{\lambda}^{\alpha}$ are nonnegative linear combinations of monomials. Therefore,

$$
C_{\lambda}^{\alpha}\left(x^{2}\right)=\sum_{\nu ;|\nu|=|\lambda|} c_{\lambda, \nu} x^{2 \nu}
$$

with coefficients $c_{\lambda, \nu} \geq 0$, and

$$
\frac{C_{\lambda}^{\alpha}\left(x^{2}\right)}{C_{\lambda}^{\alpha}(\mathbf{1})}=\sum_{\nu ;|\nu|=|\lambda|} \tilde{c}_{\lambda, \nu} x^{2 \nu}
$$

with suitable $\tilde{c}_{\lambda, \nu} \geq 0$ where $\sum_{\nu ;|\nu|=|\lambda|} \tilde{c}_{\lambda, \nu}=1$. As $C_{\lambda}^{\alpha}\left(y^{2}\right) \geq 0$ and $\sum_{|\lambda|=m} C_{\lambda}^{\alpha}\left(y^{2}\right)=|y|^{2 m}$, we conclude that

$$
\begin{aligned}
\sum_{\lambda ;|\lambda|=m} \frac{C_{\lambda}^{\alpha}\left(x^{2}\right) C_{\lambda}^{\alpha}\left(y^{2}\right)}{C_{\lambda}^{\alpha}(\mathbf{1})} & =\sum_{\lambda, \nu ;|\nu|=|\lambda|=m} \tilde{c}_{\lambda, \nu} x^{2 \nu} \cdot C_{\lambda}^{\alpha}\left(y^{2}\right) \leq|x|^{2 m} \cdot \sum_{\lambda, \nu ;|\nu|=|\lambda|=m} \tilde{c}_{\lambda, \nu} C_{\lambda}^{\alpha}\left(y^{2}\right) \\
& =|x|^{2 m} \cdot \sum_{\lambda ;|\lambda|=m} C_{\lambda}^{\alpha}\left(y^{2}\right)=|x|^{2 m}|y|^{2 m}
\end{aligned}
$$

as claimed.

Lemma 2. Let $\lambda=\left(\lambda_{1}, \ldots, \lambda_{N}\right) \geq 0$ be a partition, and choose $k_{2} \geq 0$ and $k_{1} \geq k_{2}(N-1)$. Then for $\mu:=k_{1}+k_{2}(N-1)+1 / 2$, the Pochhammer symbol $(\mu)_{\lambda}:=(\mu)_{\lambda}^{1 / k_{2}}$ satisfies

$$
\left|1-\frac{\mu^{|\lambda|}}{(\mu)_{\lambda}}\right| \leq \frac{1}{3} 2^{N(N-1)\left(k_{2}+1\right) / 2} \cdot\left(1+k_{2}(N-1)\right) \cdot \frac{|\lambda|^{2}}{k_{1}} .
$$

Proof. Consider $(\mu)_{\lambda}=\prod_{j=1}^{N}\left(\mu-k_{2}(j-1)\right)_{\lambda_{j}}$. In this $|\lambda|$-fold product, each factor can be estimated below by $\mu-k_{2}(N-1)=k_{1}+1 / 2 \geq \mu / 2$ due to our assumptions. Moreover, precisely

$$
(0+1+\cdots+(N-1))\left\lceil k_{2}\right\rceil=\frac{N(N-1)}{2} \cdot\left\lceil k_{2}\right\rceil=: r
$$

of these factors are smaller than $\mu$. We thus conclude that

$$
(\mu)_{\lambda} \geq(\mu / 2)^{r} \mu^{|\lambda|-r} \geq 2^{-N(N-1)\left(k_{2}+1\right) / 2} \cdot \mu^{|\lambda|},
$$

and thus

$$
\mu^{|\lambda|} /(\mu)_{\lambda} \leq 2^{N(N-1)\left(k_{2}+1\right) / 2} .
$$


We next prove by induction on the length $|\lambda|$ that for $k_{1} \geq k_{2}(N-1)$,

$$
\left|1-\frac{\mu^{|\lambda|}}{(\mu)_{\lambda}}\right| \leq \frac{\frac{1}{3} 2^{N(N-1)\left(k_{2}+1\right) / 2}}{\mu-k_{2}(N-1)} \cdot\left(1+k_{2}(N-1)\right) \cdot|\lambda|^{2} .
$$

As $\mu-k_{2}(N-1) \geq k_{1}$, this will imply the lemma. In fact, for $k=0,1$, the left hand side of (9) is equal to zero, while the right-hand side is nonnegative. For the induction step, consider a partition $\lambda$ of length $|\lambda| \geq 2$. Then there is a partition $\tilde{\lambda}$ with $|\tilde{\lambda}|=|\lambda|-1$ for which there exists precisely one $j=1, \ldots, N$ with $\lambda_{j}=\tilde{\lambda}_{j}+1$ while all the other components are equal. Hence, if we assume the inequality to hold for $\tilde{\lambda}$ and use (8) as well as the abbreviation $c:=\frac{2}{3}\left(1+k_{2}(N-1)\right)$, we obtain

$$
\begin{aligned}
\left|1-\frac{\mu^{|\lambda|}}{(\mu)_{\lambda}}\right|= & \left|1-\frac{\mu^{|\lambda|-1}}{(\mu)_{\tilde{\lambda}}}+\frac{\mu^{|\lambda|-1}}{(\mu)_{\tilde{\lambda}}}-\frac{\mu^{|\lambda|}}{(\mu)_{\lambda}}\right| \\
\leq & \frac{c}{\mu-k_{2}(N-1)} \cdot 2^{N(N-1)\left(k_{2}+1\right) / 2-1} \cdot(|\lambda|-1)^{2} \\
& +\frac{\mu^{|\lambda|-1}}{(\mu)_{\tilde{\lambda}}} \cdot\left|1-\frac{\mu}{\mu-k_{2}(j-1)+\lambda_{j}-1}\right| \\
\leq & \frac{c}{\mu-k_{2}(N-1)} \cdot 2^{N(N-1)\left(k_{2}+1\right) / 2-1} \cdot(|\lambda|-1)^{2} \\
& +2^{N(N-1)\left(k_{2}+1\right) / 2} \cdot \frac{\left|-k_{2}(j-1)+\lambda_{j}-1\right|}{\mu-k_{2}(j-1)+\lambda_{j}-1} \\
\leq & \frac{2^{N(N-1)\left(k_{2}+1\right) / 2-1}}{\mu-k_{2}(N-1)} \cdot\left(c(|\lambda|-1)^{2}+2 k_{2}(N-1)+2|\lambda|-2\right) \\
\leq & \frac{2^{N(N-1)\left(k_{2}+1\right) / 2-1}}{\mu-k_{2}(N-1)} \cdot c|\lambda|^{2}
\end{aligned}
$$

for $|\lambda| \geq 2$. Notice that the choice of the constant $c$ is made in order to ensure that the last inequality holds for $|\lambda| \geq 2$, which is easily checked by an elementary calculation. This completes the proof.

We are now ready to prove Proposition 1.

Proof of Proposition 1. We use the power series (3) and (4) of the Dunkl-Bessel kernels of type $\mathrm{A}$ and $\mathrm{B}$ in terms of the Jack polynomials $C_{\lambda}^{\alpha}$ and the fact that the $C_{\lambda}^{\alpha}$ are homogeneous of degree $|\lambda|$. We thus obtain

$$
J_{\left(k_{1}, k_{2}\right)}^{B}(2 \sqrt{\mu} x, i y)-J_{k_{2}}^{A}\left(-x^{2}, y^{2}\right)=\sum_{\lambda \geq 0} \frac{(-1)^{|\lambda|}}{|\lambda| !}\left(\frac{\mu^{|\lambda|}}{(\mu)_{\lambda}}-1\right) \frac{C_{\lambda}^{\alpha}\left(x^{2}\right) C_{\lambda}^{\alpha}\left(y^{2}\right)}{C_{\lambda}^{\alpha}(\mathbf{1})} .
$$

As

$$
(\mu)_{(1,0, \ldots, 0)}=\mu, \quad(\mu)_{(2,0, \ldots, 0)}=\mu(\mu+1), \quad \text { and } \quad(\mu)_{(1,1,0, \ldots, 0)}=\mu\left(\mu-k_{2}\right),
$$

the coefficients for $|\lambda| \leq 1$ are zero, and we may write the above expansion as

$$
J_{\left(k_{1}, k_{2}\right)}^{B}(2 \sqrt{\mu} x, i y)-J_{k_{2}}^{A}\left(-x^{2}, y^{2}\right)=R_{2}+R_{3}
$$


with

$$
\begin{aligned}
R_{2}= & \frac{1}{2}\left(\frac{\mu^{2}}{\mu(\mu+1)}-1\right) \frac{C_{(2,0, \ldots, 0)}^{\alpha}\left(x^{2}\right) C_{(2,0, \ldots, 0)}^{\alpha}\left(y^{2}\right)}{C_{(2,0, \ldots, 0)}^{\alpha}(\mathbf{1})} \\
& +\frac{1}{2}\left(\frac{\mu^{2}}{\mu\left(\mu-k_{2}\right)}-1\right) \frac{C_{(1,1,0, \ldots, 0)}^{\alpha}\left(x^{2}\right) C_{(1,1,0, \ldots, 0)}^{\alpha}\left(y^{2}\right)}{C_{(1,1,0, \ldots, 0)}^{\alpha}(\mathbf{1})}
\end{aligned}
$$

and

$$
R_{3}=\sum_{m \geq 3} \frac{(-1)^{m}}{m !} \sum_{|\lambda|=m}\left(\frac{\mu^{m}}{(\mu)_{\lambda}}-1\right) \cdot \frac{C_{\lambda}^{\alpha}\left(x^{2}\right) C_{\lambda}^{\alpha}\left(y^{2}\right)}{C_{\lambda}^{\alpha}(\mathbf{1})} .
$$

It now follows from Lemma 1 that under our assumptions on $k_{1}$ and $k_{2}$,

$$
\left|R_{2}\right| \leq \frac{C_{2}}{\mu} \cdot \sum_{|\lambda|=2} \frac{C_{\lambda}^{\alpha}\left(x^{2}\right) C_{\lambda}^{\alpha}\left(y^{2}\right)}{C_{\lambda}^{\alpha}(\mathbf{1})} \leq C_{2} \frac{|x|^{4}|y|^{4}}{\mu}
$$

with some $C_{2}>0$. Moreover, Lemmata 2 and 1 imply that for a suitable constant $C_{3}$,

$$
\begin{aligned}
\left|R_{3}\right| & \leq C_{3} \sum_{m \geq 3} \frac{1}{m !} \frac{m^{2}}{\mu} \sum_{|\lambda|=m} \frac{C_{\lambda}^{\alpha}\left(x^{2}\right) C_{\lambda}^{\alpha}\left(y^{2}\right)}{C_{\lambda}^{\alpha}(\mathbf{1})} \leq C_{3} \sum_{m \geq 3} \frac{m^{2}}{m ! \mu}|x|^{2 m}|y|^{2 m} \\
& \leq \frac{2 C_{3}}{\mu}|x|^{4}|y|^{4} \cdot \sum_{m \geq 3} \frac{1}{(m-2) !}|x|^{2 m-2}|y|^{2 m-2} \leq \frac{2 C_{3}}{\mu}|x|^{4}|y|^{4} \cdot e^{|x|^{2}|y|^{2}} .
\end{aligned}
$$

These estimates for $R_{2}$ and $R_{3}$ immediately imply the claimed results.

\section{References}

[1] Baker T.H., Forrester P.J., The Calogero-Sutherland model and generalized classical polynomials, Comm. Math. Phys. 188 (1997), 175-216, solv-int/9608004.

[2] Baker T.H., Forrester P.J., Nonsymmetric Jack polynomials and integral kernels, Duke Math. J. 95 (1998), $1-50$, q-alg/9612003.

[3] Dunkl C.F., Xu Y., Orthogonal polynomials of several variables, Encyclopedia of Mathematics and Its Applications, Vol. 81, Cambridge University Press, Cambridge, 2001.

[4] Faraut J., Korányi A., Analysis on symmetric cones, Oxford Science Publications, The Clarendon Press, Oxford University Press, New York, 1994.

[5] Gross K., Richards D., Special functions of matrix argument I. Algebraic induction, zonal polynomials, and hypergeometric functions, Trans. Amer. Math. Soc. 301 (1987), 781-811.

[6] Helgason S., Groups and geometric analysis. Integral geometry, invariant differential operators, and spherical functions, Pure and Applied Mathematics, Vol. 113, Academic Press, Inc., Orlando, FL, 1984.

[7] Herz C.S., Bessel functions of matrix argument, Ann. of Math. (2) 61 (1955), 474-523.

[8] Kaneko J., Selberg integrals and hypergeometric functions associated with Jack polynomials, SIAM J. Math. Anal. 24 (1993), 1086-1100.

[9] Knop F., Sahi S., A recursion and combinatorial formula for Jack polynomials, Invent. Math. 128 (1997), 9-22, q-alg/9610016.

[10] Opdam E.M., Dunkl operators, Bessel functions and the discriminant of a finite Coxeter group, Compositio Math. 85 (1993), 333-373.

[11] Rösler M., Dunkl operators: theory and applications, in Orthogonal Polynomials and Special Functions (Leuven, 2002), Editors E. Koelink et al., Springer Lect. Notes Math., Vol. 1817, Springer, Berlin, 2003, 93-135, math.CA/0210366. 
[12] Rösler M., A positive radial product formula for the Dunkl kernel, Trans. Amer. Math. Soc. 355 (2003), 2413-2438, math.CA/0210137.

[13] Rösler M., Bessel convolutions on matrix cones, Compos. Math. 143 (2007), 749-779, math.CA/0512474.

[14] Rösler M., Voit M., Limit theorems for radial random walks on $p \times q$ matrices as $p$ tends to infinity, Math. Nachr., to appear, math.CA/0703520.

[15] Stanley R.P., Some combinatorial properties of Jack symmetric functions, Adv. Math. 77 (1989), 76-115.

[16] Voit M., A limit theorem for isotropic random walks on $\mathbb{R}^{d}$ for $d \rightarrow \infty$, Russian J. Math. Phys. 3 (1995), $535-539$.

[17] Watson G.N., A treatise on the theory of Bessel functions, Cambridge University Press, Cambridge, 1966. 